\title{
Distributions of Points and Large Convex Hulls of $k$ Points
}

\author{
Hanno Lefmann \\ Fakultät für Informatik, TU Chemnitz, D-09107 Chemnitz, Germany \\ lefmann@informatik.tu-chemnitz.de
}

\begin{abstract}
We consider a variant of Heilbronn's triangle problem by asking for fixed integers $d, k \geq 2$ and any integer $n \geq k$ for a distribution of $n$ points in the $d$-dimensional unit cube $[0,1]^{d}$ such that the minimum volume of the convex hull of $k$ points among these $n$ points is as large as possible. We show that there exists a configuration of $n$ points in $[0,1]^{d}$, such that, simultaneously for $j=2, \ldots, k$, the volume of the convex hull of any $j$ points among these $n$ points is $\Omega\left(1 / n^{(j-1) /(1+|d-j+1|)}\right)$. Moreover, for fixed $k \geq d+1$ we provide a deterministic polynomial time algorithm, which finds for any integer $n \geq k$ a configuration of $n$ points in $[0,1]^{d}$, which achieves, simultaneously for $j=d+1, \ldots, k$, the lower bound $\Omega\left(1 / n^{(j-1) /(1+|d-j+1|)}\right)$ on the minimum volume of the convex hull of any $j$ among the $n$ points.
\end{abstract}

\section{Introduction}

For integers $n \geq 3$, Heilbronn's problem asks for the supremum $\Delta_{2}(n)$ of the minimum area of a triangle formed by three of $n$ points over all distributions of $n$ points in the unit square $[0,1]^{2}$. It has been observed by Erdős, see [16], that $\Delta_{2}(n)=\Omega\left(1 / n^{2}\right)$, which can be seen by considering for primes $n$ the points $P_{k}=1 / n \cdot\left(k \bmod n, k^{2} \bmod n\right), k=0,1, \ldots, n-1$. Komlós, Pintz and Szemerédi [10] improved this lower bound to the currently known best lower bound $\Delta_{2}(n)=$ $\Omega\left(\log n / n^{2}\right)$, see [4] for a deterministic polynomial time algorithm achieving this lower bound. Upper bounds were given in a series of papers by Roth [16-19] and Schmidt [20], and the currently known best upper bound is due to Komlós, Pintz and Szemerédi [9], who proved that $\Delta_{2}(n)=O\left(2^{c \sqrt{\log n}} / n^{8 / 7}\right)$ for some constant $c>0$. We remark that for $n$ points, which are chosen uniformly at random in $[0,1]^{2}$, the expected value of the minimum area of a triangle is $\Theta\left(1 / n^{3}\right)$, as was shown recently by Jiang, Li and Vitany [8].

A variant of Heilbronn's problem in dimension $d \geq 2$, which has been considered by Barequet, asks for the supremum $\Delta_{d+1, d}(n)$ - over all distributions of $n$ points in the $d$-dimensional unit cube $[0,1]^{d}$ - of the minimum volume of a $(d+1)$-point simplex among $n$ points. Barequet showed in [2] the lower bound $\Delta_{d+1, d}(n)=\Omega\left(1 / n^{d}\right)$ for fixed $d \geq 2$, see [3] for an on-line version for dimensions $d=3,4$. His lower bound was improved in [11] to $\Delta_{d+1, d}(n)=\Omega\left(\log n / n^{d}\right)$, and in [15] for dimension $d=3$ a deterministic polynomial time algorithm was given, which achieves $\Delta_{4,3}(n)=\Omega\left(\log n / n^{3}\right)$. Recently, Brass [5] improved the upper bound $\Delta_{d+1, d}(n)=O(1 / n)$ to $\Delta_{d+1, d}(n)=O\left(1 / n^{(2 d+1) /(2 d)}\right)$ for odd $d \geq 3$. 
Here we consider the following generalization of Heilbronn's problem: for fixed integers $d, k \geq 2$ and any integer $n \geq k$ find $n$ points in the $d$-dimensional unit cube $[0,1]^{d}$, such that the minimum volume of the convex hull of any $k$ points among these $n$ points is as large as possible. Let the corresponding supremum values - over all distributions of $n$ points in $[0,1]^{d}-$ on the minimum volumes of the convex hull of $k$ points among $n$ points be denoted by $\Delta_{k, d}(n)$.

This problem has been investigated also by Chazelle, who considered it in connection with lower bounds on the query complexity of range searching problems. He proved in [7] that for any fixed dimension $d \geq 2$ there exists a constant $c>0$ such that a random set of $n$ points in the unit cube $[0,1]^{d}$ satisfies with probability greater than $1-1 / n$, that the volume of the convex hull of any $k \geq \log n$ points is $\Omega(k / n)$, indeed it holds $\Delta_{k, d}(n)=\Theta(k / n)$ for $\log n \leq k \leq n$ for fixed $d \geq 2$. An extension of the range of $k$ might also improve his lower bounds on the query complexity, see [7].

Here we consider the case of fixed values $k$ and $d$. Areas of triangles arising from $n$ points in $[0,1]^{d}$ have been investigated in [12], where for fixed dimension $d \geq 2$ it has been shown that $\Delta_{3, d}(n)=\Omega\left((\log n)^{1 /(d-1)} / n^{2 /(d-1)}\right)$ and $\Delta_{3, d}(n)=O\left(1 / n^{2 / d}\right)$. Moreover, for fixed $k \leq d+1$ it has been proved recently in [14] that $\Delta_{k, d}(n)=\Omega\left((\log n)^{1 /(d-k+2)} / n^{(k-1) /(d-k+2}\right)$. For the special case of dimension $d=2$ and arbitrary $k \geq 3$ it was shown in [13] that $\Delta_{k, 2}(n)=\Omega\left((\log n)^{1 /(k-1)} / n^{(k-1) /(k-2}\right)$.

Here we prove the following lower bounds, in particular for $k>d$.

Theorem 1. Let $d, k \geq 2$ be fixed integers.

(i) Then, for any integer $n \geq k$ there exists a configuration of $n$ points in the unit cube $[0,1]^{d}$, such that, simultaneously for $j=2, \ldots, k$, the volume of the convex hull of any $j$ points among these $n$ points is

$$
\Omega\left(1 / n^{(j-1) /(1+|d-j+1|)}\right) .
$$

(ii) Moreover, for fixed $k \geq d+1$ there is a deterministic polynomial time algorithm, which finds for any integer $n \geq k$ a configuration of $n$ points in $[0,1]^{d}$, which, simultaneously for $j=d+1, \ldots, k$, achieves the lower bound $\Omega\left(1 / n^{(j-1) /(1+|d-j+1|)}\right)$ on the volume of the convex hull of any $j$ among the $n$ points in $[0,1]^{d}$.

Our arguments remain valid if $d$ and $k$ are functions of $n$, but then the lower bound (1) will depend on $d$ and $j$. Notice that for fixed integers $d, j \geq 2$, Theorem 1 yields $\Delta_{j, d}=\Omega\left(1 / n^{(j-1) /(1+|d-j+1|)}\right)$. Concerning upper bounds, for fixed integers $d, j \geq 2$ a partition of $[0,1]^{d}$ into $d$-dimensional subcubes each of volume $\Theta\left(n^{-1 / j}\right)$, yields $\Delta_{j, d}(n)=O\left(1 / n^{(j-1) / d}\right)$ for $j \leq d+1$ and $\Delta_{j, d}(n)=O(1 / n)$ for $j \geq d+1$. Moreover, for even integers $j, 2 \leq j \leq d+1$, the upper bound can be improved to $\Delta_{j, d}(n)=O\left(1 / n^{(j-1) / d+(j-2) /(2 d(d-1))}\right)$, see [14].

Somewhat surprisingly, achieving by a deterministic polynomial time algorithm for the same $n$ points in $[0,1]^{d}$ the lower bound $\Delta_{j, d}(n)=\Omega\left(1 / n^{(j-1) /(1+|d-j+1|)}\right)$, simultaneously for $j=2, \ldots, k$, where $d, k \geq 2$ are fixed integers, causes so far some difficulties w.r.t. the lower dimensional simplices, i.e., for $4 \leq j \leq d$. 


\section{Lower Bounds}

Let dist $\left(P_{i}, P_{j}\right)$ be the Euclidean distance between the points $P_{i}, P_{j} \in[0,1]^{d}$. A simplex given by the points $P_{1}, \ldots, P_{j} \in[0,1]^{d}, 2 \leq j \leq d+1$, is the set of all points $P_{1}+\sum_{i=2}^{j} \lambda_{i} \cdot\left(P_{i}-P_{1}\right)$ with $\sum_{i=2}^{j} \lambda_{i} \leq 1$ and $\lambda_{2}, \ldots, \lambda_{j} \geq 0$. The $((j-1)$ dimensional) volume of a simplex given by $j$ points $P_{1}, \ldots, P_{j} \in[0,1]^{d}, 2 \leq j \leq$ $d+1$, is defined by vol $\left(P_{1}, \ldots, P_{j}\right):=1 /(j-1) ! \cdot \prod_{i=2}^{j} \operatorname{dist}\left(P_{i} ;\left\langle P_{1}, \ldots, P_{i-1}\right\rangle\right)$, where dist $\left(P_{i} ;\left\langle P_{1}, \ldots, P_{i-1}\right\rangle\right)$ is the Euclidean distance of the point $P_{i}$ from the affine real space $\left\langle P_{1}, \ldots, P_{i-1}\right\rangle$ generated by the vectors $P_{2}^{\top}-P_{1}^{\top}, \ldots, P_{i-1}^{\top}-P_{1}^{\top}$ attached at $P_{1}$. For $j$ points $P_{1}, \ldots, P_{j} \in[0,1]^{d}, j \geq d+1$, let vol $\left(P_{1}, \ldots, P_{j}\right)$ be the ( $d$-dimensional) volume of the convex hull of the points $P_{1}, \ldots, P_{j}$.

First we prove part (i) of Theorem 1.

Proof. Let $d, k \geq 2$ be fixed integers. For arbitrary integers $n \geq k$, we select uniformly at random and independently of each other $N:=k \cdot n$ points $P_{1}, P_{2}, \ldots, P_{N}$ from the unit cube $[0,1]^{d}$.

Set $v_{j}:=\beta_{j} / n^{\gamma_{j}}$ for constants $\beta_{j}, \gamma_{j}>0, j=2, \ldots, k$, which will be fixed later. Let $V:=\left\{P_{1}, P_{2}, \ldots, P_{N}\right\}$ be the random set of chosen points in $[0,1]^{d}$. For $j=2, \ldots, k$, let $\mathcal{E}_{j}$ be the set of all $j$-element subsets $\left\{P_{i_{1}}, \ldots, P_{i_{j}}\right\} \in$ $[V]^{j}$ of points in $V$ such that vol $\left(P_{i_{1}}, \ldots, P_{i_{j}}\right) \leq v_{j}$. We estimate the expected numbers $E\left(\left|\mathcal{E}_{j}\right|\right)$ of $j$-element sets in $\mathcal{E}_{j}, j=2, \ldots, k$, and we show that for a suitable choice of the parameters $v_{2}, \ldots, v_{k}$ all numbers $E\left(\left|\mathcal{E}_{j}\right|\right)$ are not too big, i.e., $E\left(\left|\mathcal{E}_{2}\right|\right)+\cdots+E\left(\left|\mathcal{E}_{k}\right|\right) \leq(k-1) \cdot n$. Thus, there exists a choice of $N$ points $P_{1}, P_{2}, \ldots, P_{N} \in[0,1]^{d}$ such that $\left|\mathcal{E}_{2}\right|+\cdots+\left|\mathcal{E}_{k}\right| \leq(k-1) \cdot n$. Then, for $j=2, \ldots, k$, we delete one point from each $j$-element set of points in $\mathcal{E}_{j}$. The remaining points yield at least $n$ points such that the volume of the convex hull of any $j$ points of these at least $n$ points is at least $v_{j}$.

Lemma 1. Let $d, k \geq 2$ be fixed integers. For $j=2, \ldots, k$, there exist constants $c_{j, d}>0$ such that for every real $v_{j}>0$ it is

$$
E\left(\left|\mathcal{E}_{j}\right|\right) \leq c_{j, d} \cdot N^{j} \cdot v_{j}^{1+|d-j+1|} .
$$

Proof. For reals $v_{j}>0$ and random points $P_{1}, \ldots, P_{j} \in[0,1]^{d}$ we give an upper bound on the probability Prob $\left(\operatorname{vol}\left(P_{1}, \ldots, P_{j}\right) \leq v_{j}\right)$. We assume that the points $P_{1}, \ldots, P_{j}$ are numbered such that for $2 \leq g \leq h \leq j$ and $g \leq d+1$ it is

$$
\operatorname{dist}\left(P_{g} ;\left\langle P_{1}, \ldots, P_{g-1}\right\rangle\right) \geq \operatorname{dist}\left(P_{h} ;\left\langle P_{1}, \ldots, P_{g-1}\right\rangle\right) .
$$

The point $P_{1}$ can be anywhere in $[0,1]^{d}$. Given the point $P_{1}$, the probability, that the point $P_{2} \in[0,1]^{d}$ has from $P_{1}$ a Euclidean distance within the infinitesimal range $\left[r_{1}, r_{1}+d r_{1}\right]$, is at most the difference of the volumes of the $d$-dimensional balls with center $P_{1}$ and with radii $\left(r_{1}+d r_{1}\right)$ and $r_{1}$, respectively, hence

$$
\text { Prob }\left(r_{1} \leq \operatorname{dist}\left(P_{1}, P_{2}\right) \leq r_{1}+d r_{1}\right) \leq d \cdot C_{d} \cdot r_{1}^{d-1} d r_{1},
$$

where $C_{d}$ denotes the volume of the $d$-dimensional unit ball in $\mathbb{R}^{d}$. 
Given the points $P_{1}$ and $P_{2}$ with dist $\left(P_{1}, P_{2}\right)=r_{1}$, the probability that the Euclidean distance of the point $P_{3} \in[0,1]^{d}$ from the affine line $\left\langle P_{1}, P_{2}\right\rangle$ is within the infinitesimal range $\left[r_{2}, r_{2}+d r_{2}\right]$ is at most the difference of the volumes of two cylinders centered at the line $\left\langle P_{1}, P_{2}\right\rangle$ with radii $r_{2}+d r_{2}$ and $r_{2}$, respectively, and, by assumption (3), with height $2 \cdot r_{1}=2 \cdot \operatorname{dist}\left(P_{1}, P_{2}\right)$, thus

$$
\text { Prob }\left(r_{2} \leq \operatorname{dist}\left(P_{3} ;\left\langle P_{1}, P_{2}\right\rangle\right) \leq r_{2}+d r_{2}\right) \leq 2 \cdot r_{1} \cdot(d-1) \cdot C_{d-1} \cdot r_{2}^{d-2} d r_{2} .
$$

In general, let the points $P_{1}, \ldots, P_{g}, g<j$ and $g<d+1$, be given with $\operatorname{dist}\left(P_{x} ;\left\langle P_{1}, \ldots, P_{x-1}\right\rangle\right)=r_{x-1}$ for $x=2, \ldots, g$. For $g \leq j-2$ and $g \leq d-1$, by (3) the projection of the point $P_{g+1}$ onto the affine space $\left\langle P_{1}, \ldots, P_{g}\right\rangle$ is contained in a $(g-1)$-dimensional box with volume $2^{g-1} \cdot r_{1} \cdots r_{g-1}$, hence

$$
\begin{aligned}
& \text { Prob }\left(r_{g} \leq \operatorname{dist}\left(P_{g+1} ;\left\langle P_{1}, \ldots, P_{g}\right\rangle\right) \leq r_{g}+d r_{g}\right) \\
\leq & 2^{g-1} \cdot r_{1} \cdots r_{g-1} \cdot(d-g+1) \cdot C_{d-g+1} \cdot r_{g}^{d-g} d r_{g} .
\end{aligned}
$$

For $g=j-1<d$, to satisfy $\operatorname{vol}\left(P_{1}, \ldots, P_{j}\right) \leq v_{j}$, we must have $1 /(j-$ $1) ! \cdot \prod_{i=2}^{j} \operatorname{dist}\left(P_{i} ;\left\langle P_{1}, \ldots, P_{i-1}\right\rangle\right) \leq v_{j}$. By $(3)$ the projection of the point $P_{j}$ onto the affine space $\left\langle P_{1}, \ldots, P_{j-1}\right\rangle$ is contained in a $(j-2)$-dimensional box with volume $2^{j-2} \cdot r_{1} \cdots r_{j-2}$, and the point $P_{j}$ has Euclidean distance at most $\left((j-1) ! \cdot v_{j}\right) /\left(r_{1} \cdots r_{j-2}\right)$ from the affine space $\left\langle P_{1}, \ldots, P_{j-1}\right\rangle$, which happens with probability at most

$$
2^{j-2} \cdot r_{1} \cdots r_{j-2} \cdot C_{d-j+2} \cdot\left(\frac{(j-1) ! \cdot v_{j}}{r_{1} \cdots r_{j-2}}\right)^{d-j+2}
$$

For $d \leq g \leq j-1$, the projection of the point $P_{g+1}$ onto the affine space $\left\langle P_{1}, \ldots, P_{d}\right\rangle$ is contained in a $(d-1)$-dimensional box with volume at most $2^{d-1} \cdot r_{1} \cdots r_{d-1}$. Since vol $\left(P_{1}, \ldots, P_{d}, P_{g+1}\right) \leq v_{j}$ by monotonicity, the point $P_{g+1}$ has Euclidean distance at most $\left(d ! \cdot v_{j}\right) /\left(r_{1} \cdots r_{d-1}\right)$ from the affine space $\left\langle P_{1}, \ldots, P_{d}\right\rangle$, which happens with probability at most

$$
2^{d-1} \cdot r_{1} \cdots r_{d-1} \cdot \frac{2 \cdot d ! \cdot v_{j}}{r_{1} \cdots r_{d-1}}=d ! \cdot 2^{d} \cdot v_{j} .
$$

Thus, for $j \leq d$ with (4) and (5) and some constants $c_{j, d}^{*}, c_{j, d}^{* *}>0$, we obtain

$$
\begin{aligned}
& \operatorname{Prob}\left(\operatorname{vol}\left(P_{1}, \ldots, P_{j}\right) \leq v_{j}\right) \\
\leq & \int_{r_{j-2}=0}^{\sqrt{d}} \cdots \int_{r_{1}=0}^{\sqrt{d}} 2^{j-2} \cdot \frac{C_{d-j+2} \cdot((j-1) !)^{d-j+2} \cdot v_{j}^{d-j+2}}{\left(r_{1} \cdots r_{j-2}\right)^{d-j+1}} \cdot \\
& \cdot \prod_{g=1}^{j-2}\left(2^{g-1} \cdot r_{1} \cdots r_{g-1} \cdot(d-g+1) \cdot C_{d-g+1} \cdot r_{g}^{d-g}\right) d r_{j-2} \ldots d r_{1} \\
\leq & c_{j, d}^{* *} \cdot v_{j}^{d-j+2} \cdot \int_{r_{j-2}=0}^{\sqrt{d}} \cdots \int_{r_{1}=0}^{\sqrt{d}} \prod_{g=1}^{j-2}\left(r_{g}^{2 j-2 g-3}\right) d r_{j-2} \ldots d r_{1} \\
\leq & c_{j, d}^{*} \cdot v_{j}^{d-j+2} \quad \text { as } 2 \cdot j-2 \cdot g-3 \geq 1 \\
= & c_{j, d}^{*} \cdot v_{j}^{1+|d-j+1|} \quad \text { as } j \leq d .
\end{aligned}
$$


Moreover, for $j=d+1, \ldots, k$, by (4) and (6) for constants $c_{j, d}^{*}, c_{j, d}^{* *}>0$ we infer

$$
\begin{aligned}
& \text { Prob }\left(\operatorname{vol}\left(P_{1}, \ldots, P_{j}\right) \leq v_{j}\right) \\
\leq & \int_{r_{d-1}=0}^{\sqrt{d}} \cdots \int_{r_{1}=0}^{\sqrt{d}}\left(d ! \cdot 2^{d} \cdot v_{j}\right)^{j-d} . \\
& \cdot \prod_{g=1}^{d-1}\left(2^{g-1} \cdot r_{1} \cdots r_{g-1} \cdot(d-g+1) \cdot C_{d-g+1} \cdot r_{g}^{d-g}\right) d r_{d-1} \ldots d r_{1} \\
\leq & c_{j, d}^{* *} \cdot v_{j}^{j-d} \cdot \int_{r_{d-1}=0}^{\sqrt{d}} \ldots \int_{r_{1}=0}^{\sqrt{d}} \prod_{g=1}^{d-1}\left(r_{g}^{2 d-2 g-1}\right) d r_{d-1} \ldots d r_{1} \\
\leq & c_{j, d}^{*} \cdot v_{j}^{j-d} \quad \text { as } 2 \cdot d-2 \cdot g-1 \geq 1 \\
= & c_{j, d}^{*} \cdot v_{j}^{1+|d-j+1|} \quad \text { as } j \geq d+1 .
\end{aligned}
$$

By (7) and (8) we have Prob $\left(\operatorname{vol}\left(P_{1}, \ldots, P_{j}\right) \leq v_{j}\right) \leq c_{j, d}^{*} \cdot v_{j}^{1+|d-j+1|}$ for constants $c_{j, d}^{*}>0, j=2, \ldots, k$. Since there are $\left(\begin{array}{c}N \\ j\end{array}\right)$ choices for $j$ out of the $N$ random points $P_{1}, \ldots, P_{N} \in[0,1]^{d}$, inequality (2) follows.

By (2) and Markov's inequality there exist $N=k \cdot n$ points $P_{1}, \ldots, P_{N}$ in the unit cube $[0,1]^{d}$ such that for $j=2, \ldots, k$ :

$$
\left|\mathcal{E}_{j}\right| \leq k \cdot c_{j, d} \cdot N^{j} \cdot v_{j}^{1+|d-j+1|} .
$$

Lemma 2. Let $d, k \geq 2$ be fixed integers. Then, for every $\beta_{j}, \gamma_{j}$ with $0<\beta_{j} \leq$ $1 /\left(c_{j, d} \cdot k^{j+1}\right)^{1 /(1+|d-j+1|)}$ and $\gamma_{j} \geq(j-1) /(1+|d-j+1|), j=2, \ldots, k$, it is

$$
\left|\mathcal{E}_{j}\right| \leq N / k \text {. }
$$

Proof. For $j=2, \ldots, k$, by (9) and using $v_{j}=\beta_{j} / n^{\gamma_{j}}$ we infer

$$
\begin{aligned}
& \left|\mathcal{E}_{j}\right| \leq N / k \\
\Longleftarrow & k \cdot c_{j, d} \cdot N^{j} \cdot v_{j}^{1+|d-j+1|} \leq N / k \\
\Longleftrightarrow & k^{j+1} \cdot c_{j, d} \cdot \beta_{j}^{1+|d-j+1|} \cdot n^{j-1-\gamma_{j}(1+|d-j+1|)} \leq 1,
\end{aligned}
$$

which holds for $j-1 \leq \gamma_{j} \cdot(1+|d-j+1|)$ and $k^{j+1} \cdot c_{j, d} \cdot \beta_{j}^{1+|d-j+1|} \leq 1$.

Fix $\gamma_{j}:=(j-1) /(1+|d-j+1|)$ and $\beta_{j}:=1 /\left(c_{j, d} \cdot k^{j+1}\right)^{1 /(1+|d-j+1|)}, j=2, \ldots, k$. By Lemma 2 we have $\left.\left|\mathcal{E}_{2}\right|+\cdots+\left|\mathcal{E}_{k}\right| \leq((k-1) / k)\right) \cdot N$. For $j=2, \ldots, k$, we discard one point from each $j$-element set in $\mathcal{E}_{j}$. Then, the set $I \subseteq V$ of remaining points contains a subset of size $N / k=n$. These $n$ points in $[0,1]^{d}$ satisfy, simultaneously for $j=2, \ldots, k$, that the volume of the convex hull of each $j$ of these $n$ points is bigger than $v_{j}=\beta_{j} / n^{(j-1) /(1+|d-j+1|)}$, which finishes the proof of part (i) and (1) in Theorem 1. 


\section{A Deterministic Algorithm}

Here we derandomize the probabilistic arguments from Section 2 to show Theorem 1, part (ii). Throughout this section, let $k \geq d+1$. Let $B^{d}(T)$ denote the $d$-dimensional ball with radius $T$ around the origin. Then $B^{d}(T) \cap \mathbb{Z}^{d}$ is the set of all points $P \in \mathbb{Z}^{d}$, which have Euclidean distance at most $T$ from the origin. To provide a deterministic polynomial time algorithm which, for any integer $n>0$, finds a configuration of $n$ points in $[0,1]^{d}$, such that the volume of the convex hull of small sets of points is large, we discretize the unit cube $[0,1]^{d}$ by considering, for $T$ large enough, but bounded from above by a polynomial in $n$, all points in $B^{d}(T) \cap \mathbb{Z}^{d}$. This set $B^{d}(T) \cap \mathbb{Z}^{d}$ will be rescaled later by the factor $T^{d}$. However, with this discretization we have to take care of degenerate sets of points, where a set $\left\{P_{1}, \ldots, P_{j}\right\} \subset[0,1]^{d}$ with $j \geq d+1$ is called degenerate, if all points $P_{1}, \ldots, P_{j}$ are contained in a $(d-1)$-dimensional affine subspace of $\mathbb{R}^{d}$, otherwise $\left\{P_{1}, \ldots, P_{j}\right\}$ is called non-degenerate.

Set $v_{j}:=\beta_{j} \cdot T^{d} / n^{(j-1) /(j-d)}$ for suitable constants $\beta_{j}>0, j=d+1, \ldots, k$, which will be fixed later. We construct for $j=d+1, \ldots, k$ two types of $j$ element edges. For points $P_{i_{1}}, \ldots, P_{i_{j}} \in B^{d}(T) \cap \mathbb{Z}^{d}$, let $\left\{P_{i_{1}}, \ldots, P_{i_{j}}\right\} \in \mathcal{E}_{j}$ if and only if vol $\left(P_{i_{1}}, \ldots, P_{i_{j}}\right) \leq v_{j}$ and $\left\{P_{i_{1}}, \ldots, P_{i_{j}}\right\}$ is not contained in a $(d-1)$ dimensional affine subspace of $\mathbb{R}^{d}$, i.e., the set $\left\{P_{i_{1}}, \ldots, P_{i_{j}}\right\}$ is non-degenerate. Moreover, let $\left\{P_{i_{1}}, \ldots, P_{i_{j}}\right\} \in \mathcal{E}_{j}^{0}$ if and only if $\left\{P_{i_{1}}, \ldots, P_{i_{j}}\right\}$ is contained in a $(d-1)$-dimensional affine subspace of $\mathbb{R}^{d}$.

To give upper bounds on these numbers $\left|\mathcal{E}_{j}\right|$ and $\left|\mathcal{E}_{j}^{0}\right|$ of $j$-element sets, $j=$ $d+1, \ldots, k$, we use lattices in $\mathbb{Z}^{d}$.

A lattice $L$ in $\mathbb{Z}^{d}$ is a subset of $\mathbb{Z}^{d}$, which is generated by all integral linear combinations of some linearly independent vectors $b_{1}, \ldots, b_{m} \in \mathbb{Z}^{d}$, hence $L=$ $\mathbb{Z} b_{1}^{\top}+\cdots+\mathbb{Z} b_{m}^{\top}$. The parameter $m=\operatorname{rank}(L)$ is called the rank of the lattice $L$, and the set $\mathcal{B}=\left\{b_{1}, \ldots, b_{m}\right\}$ is called a basis of $L$. The set $F_{\mathcal{B}}:=\left\{\sum_{i=1}^{m} \alpha_{i} \cdot b_{i} \mid\right.$ $\left.0 \leq \alpha_{i} \leq 1, i=1, \ldots, m\right\} \subseteq \mathbb{R}^{d}$ is called the fundamental parallelepiped $F_{\mathcal{B}}$ of $\mathcal{B}$, its volume is $\operatorname{vol}\left(F_{\mathcal{B}}\right):=\left(\operatorname{det}\left(G(\mathcal{B})^{\top} \cdot G(\mathcal{B})\right)\right)^{1 / 2}$, where $G(\mathcal{B}):=\left(b_{1}, \ldots, b_{m}\right)_{d \times m}$ is the $d \times m$ generator matrix of $\mathcal{B}$ (up to the ordering of the vectors). If $\mathcal{B}$ and $\mathcal{B}^{\prime}$ are two bases of a lattice $L$ in $\mathbb{Z}^{d}$, then the volumes of the fundamental parallelepipeds are equal, i.e., $\operatorname{vol}\left(F_{\mathcal{B}}\right)=\operatorname{vol}\left(F_{\mathcal{B}^{\prime}}\right)$, see $[6]$.

For integers $a_{1}, \ldots, a_{n} \in \mathbb{Z}$, which are not all equal to 0 , let $\operatorname{gcd}\left(a_{1}, \ldots, a_{n}\right)$ denote the greatest common divisor of $a_{1}, \ldots, a_{n}$. For vectors $a=$ $\left(a_{1}, \ldots, a_{d}\right)^{\top} \in \mathbb{R}^{d}$ and $b=\left(b_{1}, \ldots, b_{d}\right)^{\top} \in \mathbb{R}^{d}$ let $\langle a, b\rangle:=\sum_{i=1}^{d} a_{i} \cdot b_{i}$ be the standard scalar product. The length of a vector $a \in \mathbb{R}^{d}$ is defined by $\|a\|:=\sqrt{\langle a, a\rangle}$. For a lattice $L$ in $\mathbb{Z}^{d}$ let $\operatorname{span}(L)$ be the linear space over the reals, which is generated by the vectors in $L$. For a subset $S=\left\{P_{1}, \ldots, P_{k}\right\} \subset \mathbb{R}^{d}$ of points the rank of $S$ is the dimension of the linear space over the reals, which is generated by the vectors $P_{2}^{\top}-P_{1}^{\top}, \ldots, P_{k}^{\top}-P_{1}^{\top}$.

A vector $a=\left(a_{1}, \ldots, a_{d}\right)^{\top} \in \mathbb{Z}^{d} \backslash\left\{0^{d}\right\}$ is called primitive, if $\operatorname{gcd}\left(a_{1}, \ldots, a_{d}\right)=1$ and $a_{j}>0$ with $j=\min \left\{i \mid a_{i} \neq 0\right\}$. A lattice $L$ in $\mathbb{Z}^{d}$ is called m-maximal, if $\operatorname{rank}(L)=m$ and no other lattice $L^{\prime} \neq L$ in $\mathbb{Z}^{d}$ with $\operatorname{rank}\left(L^{\prime}\right)=m$ contains $L$ as a proper subset. There is a one-to-one correspondence between $m$-maximal lattices in $\mathbb{Z}^{d}$ and primitive vectors $a=\left(a_{1}, \ldots, a_{d}\right)^{\top} \in \mathbb{Z}^{d} \backslash\left\{0^{d}\right\}$ : 
(i) For each lattice $L$ in $\mathbb{Z}^{d}$ with $\operatorname{rank}(L)=d-1 \geq 1$ there is exactly one primitive vector $a_{L}=\left(a_{1}, \ldots, a_{d}\right)^{\top} \in \mathbb{Z}^{d} \backslash\left\{0^{d}\right\}$ with $\left\langle a_{L}, x^{\top}\right\rangle=0$ for every $x \in L$. This vector $a_{L} \in \mathbb{Z}^{d} \backslash\left\{0^{d}\right\}$ is called the primitive normal vector of the lattice $L$.

(ii) For each lattice $L^{\prime}$ in $\mathbb{Z}^{d}$ with $\operatorname{rank}\left(L^{\prime}\right)=d-1$ there is exactly one $(d-1)$ maximal lattice $L$ in $\mathbb{Z}^{d}$ with $L^{\prime} \subseteq L$.

(iii) There exists a bijection between the set of all $(d-1)$-maximal lattices $L$ in $\mathbb{Z}^{d}$ and the set of all primitive vectors $a_{L}$ in $\mathbb{Z}^{d}$.

For a $(d-1)$-maximal lattice $L$ in $\mathbb{Z}^{d}$, a residue class of $L$ is a set $L^{\prime}$ of the form $L^{\prime}=x+L$ with $x \in \mathbb{Z}^{d}$.

The proofs of Lemmas $3-6$ concerning lattices can be found in [15].

Lemma 3 ([15]). Let $L$ be a $(d-1)$-maximal lattice in $\mathbb{Z}^{d}$ with primitive normal vector $a_{L} \in \mathbb{Z}^{d}$ and with basis $\mathcal{B}$.

(i) There exists a point $v \in \mathbb{Z}^{k} \backslash L$ such that $\mathbb{Z}^{d}$ can be partitioned into the residue classes $s \cdot v+L, s \in \mathbb{Z}$, and, for each point $x \in L$, it is $\operatorname{dist}(s \cdot v+x, \operatorname{span}(L))=$ $|s| /\left\|a_{L}\right\|$.

(ii) The volume of the fundamental parallelepiped $F_{\mathcal{B}}$ fulfills $\operatorname{vol}\left(F_{\mathcal{B}}\right)=\left\|a_{L}\right\|$.

Lemma 4 ([15]). Let $d \in \mathbb{N}$ be fixed. Let $S \subseteq B^{d}(T) \cap \mathbb{Z}^{d}$ be a set of points with $\operatorname{rank}(S) \leq d-1$. Then there exists a $(d-1)$-maximal lattice $L$ of $\mathbb{Z}^{d}$ such that $S$ is contained in some residue class $L^{\prime}=v+L$ of $L$ for some $v \in \mathbb{Z}^{d}$, and $L$ has a basis $b_{1}, \ldots, b_{d-1} \in \mathbb{Z}^{d}$ with $\max _{i=1, \ldots, d-1}\left\|b_{i}\right\|=O(T)$.

The next lemma is crucial in our considerations to estimate the numbers $\left|\mathcal{E}_{j}\right|$ and $\left|\mathcal{E}_{j}^{0}\right|$ of $j$-element sets, $j=d+1, \ldots, k$.

Lemma 5 ([15]). Let $d \in \mathbb{N}$ be fixed. Let $L$ be a $(d-1)$-maximal lattice of $\mathbb{Z}^{d}$ with primitive normal vector $a_{L} \in \mathbb{Z}^{d}$, and let $\mathcal{B}=\left\{b_{1}, \ldots, b_{d-1}\right\}$ be a basis of $L$ with $\max _{i=1, \ldots, d-1}\left\|b_{i}\right\|=O(T)$. Then the following hold:

(i) The primitive normal vector $a_{L}$ satisfies $\left\|a_{L}\right\|=O\left(T^{d-1}\right)$.

(ii) For every residue class $L^{\prime}$ of $L$ it is $\left|L^{\prime} \cap B^{d}(T)\right|=O\left(T^{d-1} /\left\|a_{L}\right\|\right)$.

For integers $g, l \in \mathbb{N}$ let $r_{g}(l)$ be the number of representations $x_{1}^{2}+\cdots+x_{g}^{2}=l$ with $x_{1}, \ldots, x_{g} \in \mathbb{Z}$.

Lemma 6 ([15]). Let $g, r \in \mathbb{N}$ be fixed integers. Then, for all integers $m \in \mathbb{N}$ :

$$
\sum_{l=1}^{m} \frac{r_{g}(l)}{l^{r}}= \begin{cases}O\left(m^{g / 2-r}\right) & \text { if } g / 2-r>0 \\ O(\log m) & \text { if } g / 2-r=0 \\ O(1) & \text { if } g / 2-r<0 .\end{cases}
$$

Lemma 7. Let $d, k \geq 2$ be fixed integers with $k \geq d+1$. For $j=d+1, \ldots, k$, there exist constants $c_{j, 0}>0$, such that the numbers $\left|\mathcal{E}_{j}^{0}\right|$ of $j$-element degenerate sets of points in $B^{d}(T) \cap \mathbb{Z}^{d}$ satisfy

$$
\left|\mathcal{E}_{j}^{0}\right| \leq c_{j, 0} \cdot T^{(d-1) j+1} \cdot \log T .
$$


Proof. By Lemma 4, each degenerate $j$-element subset of points in $B^{d}(T) \cap \mathbb{Z}^{d}$ is contained in a residue class $L^{\prime}$ of some $(d-1)$-maximal lattice $L$ in $\mathbb{Z}^{d}$, and $L$ has a basis $b_{1}, \ldots, b_{d-1} \in \mathbb{Z}^{d}$ with $\left\|b_{i}\right\|=O(T), i=1, \ldots, d-1$. By Lemma $5(\mathrm{i})$, it suffices to consider all $(d-1)$-maximal lattices $L$ with primitive normal vectors $a_{L} \in \mathbb{Z}^{d}$ of length $\left\|a_{L}\right\|=O\left(T^{d-1}\right)$.

Having fixed a $(d-1)$-maximal lattice $L$ in $\mathbb{Z}^{d}$, which is determined by its primitive normal vector $a_{L} \in \mathbb{Z}^{d}$, by Lemma $3(\mathrm{i})$, there are $O\left(T \cdot\left\|a_{L}\right\|\right)$ residue classes $L^{\prime}$ of the lattice $L$ with $L^{\prime} \cap B^{d}(T) \neq \emptyset$. By Lemma 5(ii), each set $L^{\prime} \cap B^{d}(T)$ contains $O\left(T^{d-1} /\left\|a_{L}\right\|\right)$ points. From each set $L^{\prime} \cap B^{d}(T)$ we can select $j$ points in $\left(\begin{array}{c}O\left(T^{d-1} /\left\|a_{L}\right\|\right) \\ j\end{array}\right)$ ways to obtain a degenerate set of $j$ points. This implies

$$
\begin{aligned}
& \left|\mathcal{E}_{j}^{0}\right|=O\left(\sum_{a \in \mathbb{Z}^{d},\|a\|=O\left(T^{d-1}\right)} T \cdot\|a\| \cdot\left(\begin{array}{c}
T^{d-1} /\|a\| \\
j
\end{array}\right)\right) \\
= & O\left(T^{(d-1) j+1} \cdot \sum_{a \in \mathbb{Z}^{d},\|a\|=O\left(T^{d-1}\right)} \frac{1}{\|a\|^{j-1}}\right) \\
= & O\left(T^{(d-1) j+1} \cdot \sum_{l=1}^{O\left(T^{2 d-2}\right)} \frac{r_{d}(l)}{l^{(j-1) / 2}}\right)=O\left(T^{(d-1) j+1} \cdot \log T\right),
\end{aligned}
$$

since, by Lemma 6 , we have $\sum_{l=1}^{m} r_{d}(l) / l^{(j-1) / 2}=O(\log m)$ for $j=d+1$ and $\sum_{l=1}^{m} r_{d}(l) / l^{(j-1) / 2}=O(1)$ for $j=d+2, \ldots, k$.

Lemma 8. Let $d, k \geq 2$ be fixed integers with $k \geq d+1$. For $j=d+1, \ldots, k$, there exist constants $c_{j}>0$, such that the numbers $\left|\mathcal{E}_{j}\right|$ of $j$-element nondegenerate sets of points in $B^{d}(T) \cap \mathbb{Z}^{d}$ with the volume of their convex hull at most $v_{j}$, fulfill

$$
\left|\mathcal{E}_{j}\right| \leq c_{j} \cdot T^{d^{2}} \cdot v_{j}^{j-d}
$$

Proof. For $j=d+1, \ldots, k$, consider $j$ points $P_{1}, \ldots, P_{j} \in B^{d}(T) \cap \mathbb{Z}^{d}$ with vol $\left(P_{1}, \ldots, P_{j}\right) \leq v_{j}$, where $\left\{P_{1}, \ldots, P_{j}\right\}$ is non-degenerate. Let these points be numbered such that for $2 \leq g \leq h \leq j$ and $g \leq d+1$ it is

$$
\operatorname{dist}\left(P_{g} ;\left\langle P_{1}, \ldots, P_{g-1}\right\rangle\right) \geq \operatorname{dist}\left(P_{h} ;\left\langle P_{1}, \ldots, P_{g-1}\right\rangle\right) \text {. }
$$

By Lemma 4 , the points $P_{1}, \ldots, P_{d} \in B^{d}(T) \cap \mathbb{Z}^{d}$ are contained in a residue class $L^{\prime}$ of some $(d-1)$-maximal lattice $L$ in $\mathbb{Z}^{d}$ with primitive normal vector $a_{L} \in \mathbb{Z}^{d}$, where $L$ has a basis $b_{1}, \ldots, b_{d-1} \in \mathbb{Z}^{d}$ with $\left\|b_{i}\right\|=O(T)$ for $i=1, \ldots, d-1$. By Lemma $5(\mathrm{i})$, it suffices to consider all $(d-1)$-maximal lattices $L$ with primitive vectors $a_{L} \in \mathbb{Z}^{d}$ of length $\left\|a_{L}\right\|=O\left(T^{d-1}\right)$.

We fix a $(d-1)$-maximal lattice $L$ in $\mathbb{Z}^{d}$, which is determined by its primitive normal vector $a_{L} \in \mathbb{Z}^{d}$. By Lemma $3(\mathrm{i})$, there are $O\left(T \cdot\left\|a_{L}\right\|\right)$ residue classes $L^{\prime}$ of $L$ with $L^{\prime} \cap B^{d}(T) \neq \emptyset$. By Lemma $5\left(\right.$ ii), from each set $L^{\prime} \cap B^{d}(T)$ we 
can select $d$ points $P_{1}, \ldots, P_{d}$ in $\left({ }^{O\left(T^{d-1} /\left\|a_{L}\right\|\right)}\right)$ ways. By (13) we infer for the $(d-1)$-dimensional volume vol $\left(P_{1}, \ldots, P_{d}\right)>0$, as otherwise $\left\{P_{1}, \ldots, P_{j}\right\}$ is degenerate. Also by (13) the projection of each point $P_{i} \in B^{d}(T) \cap \mathbb{Z}^{d}, i=$ $d+1, \ldots, j$, onto the residue class $L^{\prime}$ is contained in a $(d-1)$-dimensional box of volume $2^{d-1} \cdot(d-1) ! \cdot \operatorname{vol}\left(P_{1}, \ldots, P_{d}\right)$, which, by Lemma 3(ii), contains at most

$$
2^{d-1} \cdot(d-1) ! \cdot 2^{d-1} \cdot \operatorname{vol}\left(P_{1}, \ldots, P_{d}\right) /\left\|a_{L}\right\|
$$

points of $L^{\prime}$, since $P_{1}, \ldots, P_{d} \in L^{\prime}$. With vol $\left(P_{1}, \ldots, P_{d}, P_{i}\right) \leq v_{j}$ it follows that dist $\left(P_{i},\left\langle P_{1}, \ldots, P_{d}\right\rangle\right) \leq d \cdot v_{j} /$ vol $\left(P_{1}, \ldots, P_{d}\right)$, and, by Lemma $3(\mathrm{i})$, each point $P_{i} \in B^{d}(T) \cap \mathbb{Z}^{d}, i=\bar{d}+1, \ldots, j$, is contained in one of at most

$$
\left\|a_{L}\right\| \cdot d \cdot v_{j} / \operatorname{vol}\left(P_{1}, \ldots, P_{d}\right)
$$

residue classes $L^{\prime \prime}$ of $L$. By (14) in each residue class $L^{\prime \prime}$ we can choose at most $(d-1) ! \cdot 2^{2 d-2} \cdot \operatorname{vol}\left(P_{1}, \ldots, P_{d}\right) /\left\|a_{L}\right\|$ points $P_{i} \in B^{d}(T) \cap \mathbb{Z}^{d}$, hence with (15) each point $P_{i}, i=d+1, \ldots, j$, can be chosen in at most $d ! \cdot 2^{2 d-2} \cdot v_{j}$ ways. Applying this to each point $P_{d+1}, \ldots, P_{j} \in B^{d} \cap \mathbb{Z}^{d}$, we infer the upper bound

$$
\begin{aligned}
& \left|\mathcal{E}_{j}\right|=O\left(\sum_{a \in \mathbb{Z}^{d},\|a\|=O\left(T^{d-1}\right)} T \cdot\|a\| \cdot\left(\begin{array}{c}
T^{d-1} /\|a\| \\
d
\end{array}\right) \cdot v_{j}^{j-d}\right) \\
= & O\left(T^{d^{2}-d+1} \cdot v_{j}^{j-d} \cdot \sum_{a \in \mathbb{Z}^{d},\|a\|=O\left(T^{d-1}\right)} \frac{1}{\|a\|^{d-1}}\right) \\
= & O\left(T^{d^{2}-d+1} \cdot v_{j}^{j-d} \cdot \sum_{l=1}^{O\left(T^{2 d-2}\right)} \frac{r_{d}(l)}{l^{(d-1) / 2}}\right)=O\left(T^{d^{2}} \cdot v_{j}^{j-d}\right),
\end{aligned}
$$

since, by Lemma 6 , we have $\sum_{l=1}^{m} r_{d}(l) / l^{(d-1) / 2}=O\left(m^{1 / 2}\right)$.

For fixed integers $d, j, k \geq 2$ the sets $\mathcal{E}_{j}$ and $\mathcal{E}_{j}^{0}$, can easily be constructed in time polynomial in $T$. Namely, by considering every $j$-element subset $S \subset B^{d}(T) \cap \mathbb{Z}^{d}$ of points, we determine all degenerate sets of $j$ points in $B^{d}(T) \cap \mathbb{Z}^{d}$ and all nondegenerate sets of $j$ points in $B^{d}(T) \cap \mathbb{Z}^{d}$ with volume of their convex hulls at most $v_{j}$ in time $O\left(T^{d j}\right)$, since there are $\left(\begin{array}{c}O\left(T^{d}\right) \\ j\end{array}\right) j$-element subsets in $B^{d}(T) \cap \mathbb{Z}^{d}$. Let $\left|B^{d}(T) \cap \mathbb{Z}^{d}\right|=C_{d}^{\prime} \cdot T^{d}$, where $C_{d}^{\prime}>0$ is a constant. We enumerate the points in $B^{d}(T) \cap \mathbb{Z}^{d}$ by $P_{1}, \ldots, P_{C_{d}^{\prime} \cdot T^{d}}$. To each point $P_{i}$ associate a parameter $p_{i} \in[0,1], i=1, \ldots, C_{d}^{\prime} \cdot T^{d}$, and define a potential function $F\left(p_{1}, \ldots, p_{C_{d}^{\prime} \cdot T^{d}}\right)$ :

$$
\begin{aligned}
& F\left(p_{1}, \ldots, p_{C_{d}^{\prime} \cdot T^{d}}\right):=2^{p C_{d}^{\prime} T^{d} / 2} \cdot \prod_{i=1}^{C_{d}^{\prime} T^{d}}\left(1-\frac{p_{i}}{2}\right)+ \\
& +\sum_{j=d+1}^{k} \frac{\sum_{\left\{i_{1}, \ldots i_{j}\right\} \in \mathcal{E}_{j}} p_{i_{1}} \cdots p_{i_{j}}}{2 \cdot k \cdot p^{j} \cdot c_{j} \cdot T^{d^{2}} \cdot v_{j}^{j-d}}+\sum_{j=d+1}^{k} \frac{\sum_{\left\{i_{1}, \ldots, i_{j}\right\} \in \mathcal{E}_{j}^{0}} p_{i_{1}} \cdots p_{i_{j}}}{2 \cdot k \cdot p^{j} \cdot c_{j, 0} \cdot T^{(d-1) j+1} \cdot \log T} .
\end{aligned}
$$


With the initialisation $p_{1}:=\cdots:=p_{C_{d}^{\prime} \cdot T^{d}}:=p=(2 \cdot k \cdot n) /\left(C_{d}^{\prime} \cdot T^{d}\right) \leq 1$, i.e., say $T^{d}=\omega(n)$, we infer by Lemmas 7 and 8 that $F(p, \ldots, p)<(2 / e)^{p C_{d}^{\prime} T^{d} / 2}+$ $(2 k-2 d) /(2 k)$, which is less than 1 for $p \cdot C_{d}^{\prime} \cdot T^{d} \geq 7 \cdot \ln k$. Using the linearity of $F\left(p_{1}, \ldots, p_{C_{d}^{\prime} \cdot T^{d}}\right)$ in each $p_{i}$, we minimize $F\left(p_{1}, \ldots, p_{C_{d}^{\prime} \cdot T^{d}}\right)$ by choosing one after the other $p_{i}:=0$ or $p_{i}:=1$ for $i=1, \ldots, C_{d}^{\prime} \cdot T^{d}$, and finally we obtain $F\left(p_{1}, \ldots, p_{C_{d}^{\prime} \cdot T^{d}}\right)<1$. With $V^{*}=\left\{P_{i} \in B^{d}(T) \cap \mathbb{Z}^{d} \mid p_{i}=1\right\}$ this yields a subset $V^{*} \subseteq B^{d}(T) \cap \mathbb{Z}^{d}$ of points and subsets $\mathcal{E}_{j}^{0 *}:=\left[V^{*}\right]^{j} \cap \mathcal{E}_{j}^{0}$ and $\mathcal{E}_{j}^{*}:=\left[V^{*}\right]^{j} \cap \mathcal{E}_{j}$ of $j$-element sets, $j=d+1, \ldots, k$, such that

$$
\begin{aligned}
\left|V^{*}\right| & \geq p \cdot C_{d}^{\prime} \cdot T^{d} / 2 \\
\left|\mathcal{E}_{j}^{*}\right| & \leq 2 \cdot k \cdot p^{j} \cdot c_{j} \cdot T^{d^{2}} \cdot v_{j}^{j-d} \\
\left|\mathcal{E}_{j}^{0 *}\right| & \leq 2 \cdot k \cdot p^{j} \cdot c_{j, 0} \cdot T^{(d-1) j+1} \cdot \log T .
\end{aligned}
$$

By choice of the parameters $v_{j}, j=d+1, \ldots, k$, the running time of this derandomization is $O\left(T^{d}+\sum_{j=d+1}^{k}\left(\left|\mathcal{E}_{j}\right|+\left|\mathcal{E}_{j}^{0}\right|\right)\right)=O\left(T^{d k}\right)$, which is polynomial in $T$ for fixed integers $d, k \geq 2$.

Lemma 9. For $j=d+1, \ldots, k$, and $0<\beta_{j} \leq\left(C_{d}^{\prime j} /\left(2^{j+2} \cdot k^{j+1} \cdot c_{j, d}\right)\right)^{1 /(j-d)}$, it is

$$
\left|\mathcal{E}_{j}^{*}\right| \leq\left|V^{*}\right| /(2 \cdot k) .
$$

Proof. By (16) and (17) with $v_{j}:=\beta_{j} \cdot T^{d} / n^{\frac{j-1}{j-d}}$, and $p=(2 \cdot k \cdot n) /\left(C_{d}^{\prime} \cdot T^{d}\right)$, and with $\beta_{j}>0$ it is

$$
\begin{aligned}
& \left|\mathcal{E}_{j}^{*}\right| \leq\left|V^{*}\right| /(2 \cdot k) \\
\Longleftarrow & 2 \cdot k \cdot p^{j} \cdot c_{j} \cdot T^{d^{2}} \cdot v_{j}^{j-d} \leq p \cdot C_{d}^{\prime} \cdot T^{d} /(4 \cdot k) \\
\Longleftrightarrow & 8 \cdot k^{2} \cdot\left(\frac{2 \cdot k \cdot n}{C_{d}^{\prime} \cdot T^{d}}\right)^{j-1} \cdot c_{j} \cdot T^{d^{2}-d} \cdot\left(\frac{\beta_{j} \cdot T^{d}}{n^{j-1}}\right)^{j-d} \leq C_{d}^{\prime} \\
\Longleftrightarrow & 2^{j+2} \cdot k^{j+1} \cdot c_{j} \cdot \beta_{j}^{j-d} \leq C_{d}^{j},
\end{aligned}
$$

which holds for $\beta_{j}^{j-d} \leq C_{d}^{\prime j} /\left(2^{j+2} \cdot k^{j+1} \cdot c_{j}\right), j=d+1, \ldots, k$.

Lemma 10. For $j=d+1, \ldots, k$, and $T /(\log T)^{1 /(j-1)}=\omega(n)$, it is

$$
\left|\mathcal{E}_{j}^{0 *}\right| \leq\left|V^{*}\right| /(2 \cdot k) .
$$

Proof. By (16) and (18), with $p=(2 \cdot k \cdot n) /\left(C_{d}^{\prime} \cdot T^{d}\right), j=d+1, \ldots, k$, we infer

$$
\begin{aligned}
& \left|\mathcal{E}_{j}^{0 *}\right| \leq\left|V^{*}\right| /(2 \cdot k) \\
\Longleftarrow & 2 \cdot k \cdot p^{j} \cdot c_{j, 0} \cdot T^{(d-1) j+1} \cdot \log T \leq p \cdot C_{d}^{\prime} \cdot T^{d} /(4 \cdot k) \\
\Longleftrightarrow & 8 \cdot k^{2} \cdot\left(\frac{2 \cdot k \cdot n}{C_{d}^{\prime} \cdot T^{d}}\right)^{j-1} \cdot c_{j, 0} \cdot T^{(d-1) j-d+1} \cdot \log T \leq C_{d}^{\prime} \\
\Longleftrightarrow & 2^{j+2} \cdot k^{j+1} \cdot c_{j, 0} \cdot \frac{n^{j-1}}{T^{j-1}} \cdot \log T \leq C_{d}^{\prime j},
\end{aligned}
$$

which holds for $T /(\log T)^{1 /(j-1)}=\omega(n)$. 
With $T:=n \cdot \log n$ and $\beta_{j}:=\left(C_{d}^{\prime j} /\left(2^{j+2} \cdot k^{j+1} \cdot c_{j}\right)^{1 /(j-d)}, j=d+1, \ldots, k\right.$, the assumptions of Lemmas 9 and 10 are fulfilled. By deleting in time $O\left(\left|V^{*}\right|+\right.$ $\left.\sum_{j=d+1}^{k}\left(\left|\mathcal{E}_{j}^{*}\right|+\left|\mathcal{E}_{j}^{0 *}\right|\right)\right) O\left(T^{k d}\right)$ one point from each $j$-element set in $\mathcal{E}_{j}^{*}$ and $\mathcal{E}_{j}^{0 *}$, $j=d+1, \ldots, k$, the remaining points yield a subset $V^{* *} \subseteq V^{*}$ of size at least $\left|V^{*}\right| / k \geq p \cdot C_{d}^{\prime} \cdot T^{d} /(2 \cdot k)=n$. Then these at least $n$ points in $B^{d}(T) \cap \mathbb{Z}^{d}$ satisfy that the volume of the convex hull of any $j$ of these points, $j=d+1, \ldots, k$, is at least $v_{j}$, i.e., $\Omega\left(T^{d} / n^{(j-1) /(j-d)}\right)$. After rescaling by the factor $T^{d}$, we have at least $n$ points in the unit cube $[0,1]^{d}$ such that the volume of the convex hull of any $j$ of these points is $\Omega\left(1 / n^{(j-1) /(j-d)}\right), j=d+1, \ldots, k$. Altogether the running time of this deterministic algorithm is $O\left((n \cdot \log n)^{d k}\right)$ for fixed $d, k \geq 2$, hence polynomial in $n$, which finishes the proof of Theorem 1, part (ii).

\section{Concluding Remarks}

Our arguments yield a deterministic polynomial time algorithm for obtaining a distribution of $n$ points in $[0,1]^{d}$, which, for fixed integers $j \geq d+1$, shows $\Delta_{j, d}(n)=\Omega\left(1 / n^{(j-1) /(1+|d-j+1|)}\right)$. With the results from [14], i.e., using a result of Ajtai, Komlós, Pintz, Spencer and Szemerédi [1] on uncrowded hypergraphs we can improve Theorem 1 slightly (Details are omitted here), namely for fixed integers $d, k \geq 3$ and a fixed integer $j_{0}$ with $3 \leq j_{0} \leq d+1$ one can find in polynomial time a configuration of $n$ points in $[0,1]^{d}$, such that, simultaneously for $j=2, \ldots, k$ but $j \neq j_{0}$, the volume of the convex hull of $j$ points among these $n$ points is at least $\Omega\left(1 / n^{(j-1) /(1+|d-j+1|)}\right)$ and $\Delta_{j_{0}, d}(n)=\Omega\left((\log n)^{1 /\left(d-j_{0}+2\right)} / n^{\left(j_{0}-1\right) /\left(d-j_{0}+2\right.}\right)$. It would be interesting to get such an improvement by a logarithmic factor for the same $n$ points in $[0,1]^{d}$, simultaneously for $3 \leq j \leq k$, for fixed $d, k$.

Moreover, improvements of the existing upper bounds, which were given in the introduction, are desirable. Also investigations of this problem for non-constant values of $k$ might be of interest in view of the results of Chazelle [7].

\section{References}

1. M. Ajtai, J. Komlós, J. Pintz, J. Spencer and E. Szemerédi, Extremal Uncrowded Hypergraphs, Journal of Combinatorial Theory Ser. A, 32, 1982, 321-335.

2. G. Barequet, A Lower Bound for Heilbronn's Triangle Problem in d Dimensions, SIAM Journal on Discrete Mathematics 14, 2001, 230-236.

3. G. Barequet, The On-Line Heilbronn's Triangle Problem in Three and Four Dimensions, Proceedings '8rd Annual International Computing and Combinatorics Conference COCOON'02', LNCS 2387, Springer, 2002, 360-369.

4. C. Bertram-Kretzberg, T. Hofmeister and H. Lefmann, An Algorithm for Heilbronn's Problem, SIAM Journal on Computing 30, 2000, 383-390.

5. P. Brass, An Upper Bound for the d-Dimensional Heilbronn Triangle Problem, SIAM Journal on Discrete Mathematics 19, 192-195, 2005.

6. J. W. S. Cassels, An Introduction to the Geometry of Numbers, Vol. 99, SpringerVerlag, New York, 1971. 
7. B. Chazelle, Lower Bounds on the Complexity of Polytope Range Searching, Journal of the American Mathematical Society 2, 637-666, 1989.

8. T. Jiang, M. Li and P. Vitany, The Average Case Area of Heilbronn-type Triangles, Random Structures \& Algorithms 20, 2002, 206-219.

9. J. Komlós, J. Pintz and E. Szemerédi, On Heilbronn's Triangle Problem, Journal of the London Mathematical Society, 24, 1981, 385-396.

10. J. Komlós, J. Pintz and E. Szemerédi, A Lower Bound for Heilbronn's Problem, Journal of the London Mathematical Society, 25, 1982, 13-24.

11. H. Lefmann, On Heilbronn's Problem in Higher Dimension, Combinatorica 23, 2003, 669-680.

12. H. Lefmann, Large Triangles in the d-Dimensional Unit-Cube, Proceedings 10th Annual International Conference Computing and Combinatorics COCOON'04, eds. K.-Y. Chwa and J. I. Munro, LNCS 3106, Springer, 2004, 43-52.

13. H. Lefmann, Distributions of Points in the Unit-Square and Large $k$-Gons, Proceedings ACM-SIAM Syposium on Discrete Algorithms, SODA'05, ACM und SIAM, 241-250, 2005.

14. H. Lefmann, Large Simplices in the d-Dimensional Unit-Cube (Extended Abstract), Proceedings 11th Annual International Conference Computing and Combinatorics COCOON'05, ed. L. Wang, LNCS 3595, Springer, 2005, 514-523.

15. H. Lefmann and N. Schmitt, A Deterministic Polynomial Time Algorithm for Heilbronn's Problem in Three Dimensions, SIAM Journal on Computing 31, 2002, 1926-1947.

16. K. F. Roth, On a Problem of Heilbronn, Journal of the London Mathematical Society 26, 1951, 198-204.

17. K. F. Roth, On a Problem of Heilbronn, II, and III, Proc. of the London Mathematical Society (3), 25, 1972, 193-212, and 543-549.

18. K. F. Roth, Estimation of the Area of the Smallest Triangle Obtained by Selecting Three out of $n$ Points in a Disc of Unit Area, Proc. of Symposia in Pure Mathematics, 24, 1973, AMS, Providence, 251-262.

19. K. F. Roth, Developments in Heilbronn's Triangle Problem, Advances in Mathematics, 22, 1976, 364-385.

20. W. M. Schmidt, On a Problem of Heilbronn, Journal of the London Mathematical Society (2), 4, 1972, 545-550. 